\title{
Bioinformatics analysis of the structure and function of NADPH-cytochrome p450 reductase of Plasmodium vivax
}

\author{
ZHIGANG FAN ${ }^{1,2^{*}},{\text { KAIJIE } \text { LI }^{3 *}, \text { LINGMIN ZHANG }^{4}, \text { FAN CHEN }^{5} \text {, QIANG WU }}^{1}$, NA LI ${ }^{1}$, \\ SAIFENG ZHONG ${ }^{1}$, GUIFEN LIN ${ }^{1}$ and GUOGANG YAN ${ }^{6}$ \\ ${ }^{1}$ School of Tropical and Laboratory Medicine; ${ }^{2}$ Hainan Provincial Key Laboratory of Tropical Medicine, \\ Hainan Medical College, Haikou 571199; ${ }^{3}$ Hubei Provincial Center for Disease Control and Prevention, Wuhan 430079; \\ ${ }^{4}$ Department of Parasitology, Medical College, Jinan University, Guangzhou 510623; \\ ${ }^{5}$ Department of Biochemistry and Molecular Biology, Medical School, Wuhan University, \\ Wuhan 430072; ${ }^{6}$ School of Nursing, Hainan Medical College, Haikou 571199, P.R. China
}

Received December 15, 2012; Accepted February 5, 2013

DOI: $10.3892 /$ br.2013.71

\begin{abstract}
The structure of NADPH-cytochrome p450 reductase (CPR) of Plasmodium falciparum (P. falciparum or Pf) has been determined using bioinformatics analysis. However, that of Plasmodium vivax (P. vivax or Pv) has not yet been determined. This study aimed to analyze the structure and function of PvCPR using bioinformatics analysis. The results demonstrated that PVCPR was an unstable and alkaline enzyme located in the cytoplasm of parasites with a signal peptide. It possessed seven types of signal sites and eight protein-protein binding sites, and had a tertiary structure resembling a forceps with a single wing, which differed from that of PfCPR. It also had nine linear B-cell epitopes and 10 antigenicity sites, which were not homologous with the amino acid sequence of Homo sapiens (H. sapiens or Hs) CPR and six fragments that were similar to fragments of immune-related protein sequences from $H$. sapiens. Therefore, the function of
\end{abstract}

Correspondence to: Dr Zhigang Fan, School of Tropical and Laboratory Medicine, Hainan Medical College, 3 Xueyuan Road, Haikou 571199, P.R. China

E-mail: yuyanghoutu2006@yahoo.com.cn

Dr Guogang Yan, School of Nursing, Hainan Medical College, 3 Xueyuan Road, Haikou 571199, P.R. China

E-mail: yanguogang8@yahoo.com.cn

*Contributed equally

Abbreviations: $P$. vivax or $\mathrm{Pv}$, Plasmodium vivax; $P$. falciparum or Pf, Plasmodium falciparum; H. sapiens or Hs, Homo sapiens; CPR, NADPH-cytochrome p450 reductase; NOR, nuclear organizer region; TLR, toll-like receptor

Key words: NADPH-cytochrome p450 reductase, Plasmodium falciparum, Plasmodium vivax, bioinformatics, linear B-cell epitope, antigenicity sites, immune escape
PvCPR may be different from that of PfCPR, and PvCPR may participate in the immune escape of $P$. vivax.

\section{Introduction}

Plasmodium vivax ( $P$. vivax or $\mathrm{Pv}$ ) malaria epidemics occur mainly on the American and Asian continents (1). P. vivax is responsible for $74 \%$ of cases on the American continent (2) and for $>90 \%$ of the cases in China (3). The function of NADPH-cytochrome p450 reductase (CPR) in mammals involves electron transport. The structure and function of Plasmodium falciparum (P. falciparum or Pf) CPR have been analyzed using bioinformatics (4), while those of PvCPR have not. Therefore, the aim of this study was to investigate the structure and function of PvCPR using bioinformatics analysis.

\section{Materials and methods}

The methods used to study the amino acid (aa) sequence, physicochemical properties, subcellular localization, secondary protein structure and 3D structure have been previously described (4). The PvCPR signal peptide was predicted using the Signal P program (http:/www.cbs.dtu.dk/services/ SignalP/). Identification and comparison of similar sequences were performed using BLAST (http://blast.ncbi.nlm.nih. gov/Blast.cgi). The location of the linear B-cell epitopes and antigenicity sites were predicted with http://www. cbs.dtu.dk/ services/BepiPred/ and http://tools.immuneepitope.org/tools/ bcell/iedb_input, respectively. Identification and comparison of similar sequences were performed with http://blast.ncbi. nlm.nih.gov/Blast.cgi.

\section{Results}

Physical and chemical characteristics of PvCPR. The aa sequence of PvCPR (NCBI reference sequence: XP_001614764.1) contained 746 aa with a molecular weight of $85.175 \mathrm{kDa}$ and a predicted isoelectric point of 8.72 . The 
Table I. Linear B-cell epitopes and antigenicity sites of PvCPR.

\begin{tabular}{|c|c|c|c|c|c|c|}
\hline \multicolumn{3}{|c|}{ Linear B-cell epitopes (aa sequences) } & \multicolumn{4}{|c|}{ Antigenicity sites (aa sequences) } \\
\hline $1-3$ & $49-58$ & $67-77$ & $6-23$ & $28-38$ & $40-46$ & $60-66$ \\
\hline $117-125$ & $137-142$ & $183-193$ & $88-99$ & $106-117$ & $125-136$ & $145-152$ \\
\hline $250-251$ & $253-290$ & 314-322 & $155-175$ & 204-234 & $236-244$ & $246-254$ \\
\hline $347-349$ & $360-367$ & $376-416$ & $281-287$ & $323-335$ & $337-345$ & $369-375$ \\
\hline $498-515$ & 525 & $541-549$ & 391-397 & $401-443$ & $451-456$ & $463-468$ \\
\hline $571-577$ & 593 & $605-608$ & $474-480$ & $487-508$ & $517-536$ & $544-549$ \\
\hline 630 & $654-662$ & $673-675$ & $551-558$ & $563-571$ & $579-596$ & $607-617$ \\
\hline $708-712$ & $714-715$ & $725-730$ & $621-628$ & $634-641$ & $663-672$ & $675-706$ \\
\hline 736-737 & $740-741$ & & & & & \\
\hline
\end{tabular}

PvCPR, Plasmodium vivax NADPH-cytochrome p450 reductase.

estimated half-lives of the protein in mammals, yeast and E. coli were $30,>20$ and $>10 \mathrm{~h}$, respectively, and its instability coefficient was 42.89 , which was characteristic of an unstable protein. The grand average of hydrophobicity of PvCPR was -0.375 . Therefore, PvCPR was an unstable and alkaline enzyme.

Subcellular localization of PvCPR. The probability of localization of PvCPR was predicted to be $43.5 \%$ in the mitochondria, $17.4 \%$ in the cytoplasm, $13.0 \%$ in the vacuole, $4.3 \%$ in the Golgi apparatus, $4.3 \%$ in the endoplasmic reticulum, $4.3 \%$ in the nucleus and $13.0 \%$ outside the cell. PvCPR was predicted to localize in the mitochondria of $P$. vivax by the k-NN prediction; however, the reliability of PvCPR location in the cytoplasm was 76.7 according to Reinhardt's method for cytoplasmic/nuclear discrimination. PvCPR was predicted to consist of a signal peptide (probability of 0.719). The likely cleavage site was predicted to be located between aa 23 and 24 or between aa 24 and 25 .

Molecular evolutionary conserved domains of PvCPR. PvCPR was most closely related to $P$. knowlesi (Pk) CPR (NCBI reference sequence: XP_002258503.1) with 82\% identity and $89 \%$ similarity, but not to P. falciparum (4). PvCPR contained one complete FMN_red superfamily and one incomplete FNR_like superfamily, similar to PfCPR.

Structure of PvCPR. The secondary structure of PvCPR comprised $31.77 \% \alpha$-helices, $15.15 \% \quad \beta$-strands and $53.08 \%$ loops. In addition, this protein possessed no transmembrane region. Approximately $50 \%$ of the aa were exposed on the surface of the protein. Five N-glycosylation sites, three cAMPand cGMP-dependent protein kinase phosphorylation sites, 12 protein kinase $\mathrm{C}$ phosphorylation sites, eight casein kinase II phosphorylation sites, four tyrosine kinase phosphorylation sites, seven $\mathrm{N}$-myristoylation sites, one amidation site and eight protein-protein binding sites were identified in PvCPR. Fifteen functional sites and six protein-protein binding sites were located in the FNR_like superfamily region and one protein-protein binding site was located in the nuclear organizer region (NOR), which was predicted to span between aa 203-320.

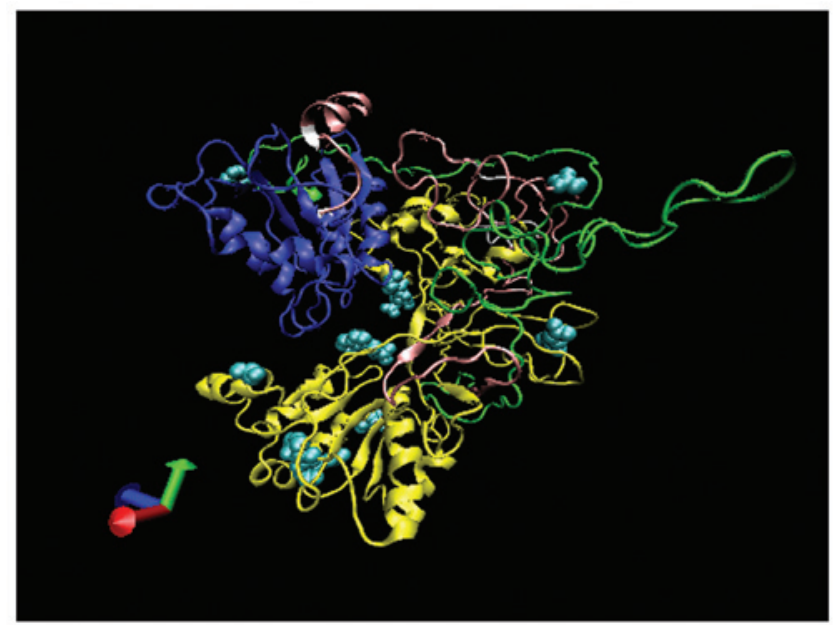

Figure 1 . The tertiary structure of Plasmodium vivax NADPH-cytochrome p450 reductase (PvCPR). Blue, sequences located in FMN_red superfamily; green, sequences located in nuclear organizer regions (NORs); yellow, sequences located in FNR_like superfamily; white, conserved sequences; pink, non-conserved sequences; cyan, protein-protein binding sites.

The tertiary structure of the protein (aa 45-746) resembled a forceps with a single wing (Fig. 1). The FMN_red superfamily and FNR_like superfamily formed a large and deep indentation. A section of the NOR (aa 253-290) formed a single wing. Of note, most of the functional sites were scattered on the surface of the protein.

Linear B-cell epitopes and antigenicity sites of PvCPR. PvCPR was located in the cytoplasm of parasites and belonged to a secretory protein. It was not known whether PvCPR was an ideal target for diagnosis, vaccines or drugs. Therefore, the linear B-cell epitopes, antigenicity sites and unique sites of PvCPR were analyzed. There were 26 linear B-cell epitopes and 32 antigenicity sites in the aa sequence of PvCPR (Table I). Nine linear B-cell epitopes were not homologous with the aa sequence of Homo sapiens (H. sapiens or Hs) CPR, as follows: aa 1-3, aa 250-251, aa 253-290, aa 314-322, aa 347-349, aa 593, aa 630 , aa $725-730$ and aa 736-737. Epitopes aa 137-142, aa 376-416, aa 571-579 and aa 654-662 were less homologous 
with those of HsCPR compared to the remaining linear B-cell epitopes. The following 10 antigenicity sites were not homologous with the aa sequence of HsCPR: aa 6-23, aa 28-38, aa 40-46, aa 204-234, aa 236-244, aa 246-254, aa 281-287, aa 323-335, aa 391-397 and aa 563-571. In the tertiary protein structure, eight linear B-cell epitopes (between aa 1-746) were located on the surface and one antigenicity site (aa 563-571), was located on the inner side of the surface, whereas six antigenicity sites (located between aa 45-746) were located on the surface.

Unique fragments among the a sequence of PvCPR. The aa fragments of PvCPR which had functional sites and no homologous fragments with the aa sequence of HsCPR included aa 1-51, aa 135-138, aa 140-147, aa 205-357, aa 366-370, aa 373-407, aa 555-577 and aa 721-730. There was no unique aa fragment that was non-homologous with other protein sequences from $H$. sapiens. Six fragments were similar to fragments of immune-related protein sequences from $H$. sapiens. These immune-related proteins included immunoglobulin heavy constant $\gamma 1$, Toll/interleukin-1 receptor-like protein 3 , toll-like receptor 5 (TLR5), interleukin-4 receptor (IL-4R) and T-cell receptor $\beta$ variable 3 .

\section{Discussion}

The physical and chemical characteristics of PvCPR were similar to those of PfCPR (4). PvCPR was located in the cytoplasm of parasites and contained a signal peptide, which differed from that of PfCPR. The tertiary structure of PvCPR was different from that of PfCPR. The changes in the protein structure during evolution may have led to loss or gain of certain functions (5). PvCPR, as opposed to PfCPR, possessed three cAMP- and cGMP-dependent protein kinase phosphorylation sites and one amidation site. However, it did not exhibit vacuole target and bileucine motifs, in contrast to PfCPR. Therefore, the function of PvCPR may differ from that of PfCPR, which may be responsible for the differences in physiology and pathogenicity between $P$. vivax and $P$. falciparum.

PvCPR had nine linear B-cell epitopes and 10 antigenicity sites, which were not homologous with the aa sequence of HsCPR. Assuming that PvCPR is the key protein of P. vivax, these sites may be ideal targets for vaccines and drugs. However, if this is not the case, these sites may be ideal diagnostic markers of $P$. vivax. In addition, six fragments of PvCPR were similar to protein fragments from $H$. sapiens, which participate in the innate and adaptive immune responses, such as IL-4R and TLR5 (6,7). Therefore, PvCPR may be involved in the immune escape of $P$. vivax.

\section{Acknowledgements}

The authors thank colleagues at the School of Tropical and Laboratory Medicine and Hainan Provincial Key Laboratory of Tropical Medicine and the teachers from the Department of Immunology of Tongji Medical College in Huazhong University of Science and Technology. This study was supported by Hainan Natural Science Fund (no. 812199) and Scientific Research Funds of Hainan Medical University in 2011 (no. 2010-014).

\section{References}

1. Mueller I, Galinski MR, Baird JK, et al: Key gaps in the knowledge of Plasmodium vivax, a neglected human malaria parasite. Lancet Infect Dis 9: 555-666, 2009.

2. Valencia SH, Rodríguez DC, Acero DL, et al: Platform for Plasmodium vivax vaccine discovery and development. Mem Inst Oswaldo Cruz 106 (Suppl 1): 179-192, 2011.

3. Zhou SS, Wang Y and Xia ZG: Malaria situation in the People's Republic Of China in 2009. Chin J Parasitol Parasit Dis 29: 1-3, 2011.

4. Fan Z, Zhang L, Yan G, et al: Bioinformatics analysis for structure and function of CPR of Plasmodium falciparum. Asian Pac J Trop Med 4: 85-87, 2011.

5. Bridgham JT, Ortlund EA and Thomton JW: An epistatic ratchet constrains the direction of glucocorticoid receptor evolution. Nature 461: 515-519, 2009.

6. Burt BM, Bader A, Winter D, et al: Expression of interleukin-4 receptor alpha in human pleural mesothelioma is associated with poor survival and promotion of tumor inflammation. Clin Cancer Res 18: 1568-1577, 2012.

7. Descamps D, Le Gars M, Balloy V, et al: Toll-like receptor 5 (TLR5), IL-1 $\beta$ secretion, and asparagine endopeptidase are critical factors for alveolar macrophage phagocytosis and bacterial killing. Proc Natl Acad Sci USA 109: 1619-1624, 2012 . 